\title{
Effects of Inflammation on Hippocampus and Substantia Nigra Responses to Novelty in Healthy Human Participants
}

\author{
Neil A Harrison ${ }^{*, 1,2}$, Mara Cercignani', Valerie Voon ${ }^{3}$ and Hugo D Critchley ${ }^{1,2}$ \\ 'Clinical Imaging Sciences Centre, Brighton and Sussex Medical School, University of Sussex Campus, Brighton, UK; ${ }^{2}$ Sackler Centre for \\ Consciousness Science, University of Sussex, Brighton, UK; ${ }^{3}$ Department of Psychiatry, University of Cambridge, Cambridge, UK
}

\begin{abstract}
Humans are naturally inquisitive. This tendency is adaptive, aiding identification of potentially valuable novel outcomes. The dopaminergic substantia nigra (SN) is implicated in the drive to explore novel stimuli and situations. However, infection and inflammation inhibit the motivation to seek out novelty. This likely serves to limit exposure to uncertain, potentially detrimental outcomes when metabolic resources are limited. Nevertheless, the neural mechanisms through which inflammation constrains novelty seeking are poorly understood. We therefore scanned 16 healthy participants (6 male, mean $27.2 \pm 7.3$ years), using $\mathrm{fMRI}$, once following experimental inflammation (intramuscular (i.m.) typhoid vaccination) and once after placebo (i.m. saline), with the aim of characterizing effects of inflammation on neural processing of novel and familiar place, and face stimuli. We specifically tested the effects of inflammation on the hypothesized roles of SN and hippocampus in novelty processing. Typhoid vaccination evoked a nearly threefold increase in circulating pro-inflammatory cytokine (interleukin-6) levels $3 \mathrm{~h}$ after injection, indicating induction of mild systemic inflammation. Enhanced hippocampal responses to novel (compared with familiar) stimuli were observed following both vaccine and placebo, consistent with intact central novelty detection. However, the normal bilateral reactivity of SN to stimulus novelty was significantly attenuated following inflammation. Correspondingly, inflammation also markedly impaired novelty-related functional coupling between the SN and hippocampus. These data extend previous findings of SN sensitivity to mild inflammation associated with changes in psychomotor responding, and suggest that inflammation-induced blunting of SN responses to hippocampal novelty signals may represent a plausible mechanism through which inflammation impairs motivational responses to novelty.
\end{abstract}

Neuropsychopharmacology (2015) 40,83I-838; doi:I0.1038/npp.2014.222; published online 24 September 2014

\section{INTRODUCTION}

Humans are innately inquisitive and demonstrate a marked preference for novelty (Berlyne, 1955). This novelty preference is common to other species (Panksepp, 1998) and may motivate environmental exploration, enabling the identification of new sources of reward (Kakade and Dayan, 2002). However, novelty exploration is not without risk. Considerable energy can be consumed investigating ultimately unrewarding novel environments or foodstuffs, and exploration may increase exposure to predators or sources of infection (Lima and Dill, 1990). Interestingly, infection itself markedly reduces novelty exploration in rodents (Kusnecov et al, 1999). This reduction is a key component of sickness behaviors, a cluster of stereotyped behavioral adaptations that support, coordinate, and prioritize wholeorganism immunological responses against the infecting organism (Hart, 1988). Pro-inflammatory cytokines including interleukin (IL)-1 can elicit sickness behaviors directly, impairing novelty preference, thereby mechanistically link-

*Correspondence: Dr NA Harrison, Clinical Imaging Sciences Centre, Brighton and Sussex Medical School, University of Sussex, Falmer, Brighton BNI 9RR, UK, Tel: +44 01273 876657, Fax: +44 0 I273 87672I,

E-mail: n.harrison@bsms.ac.uk

Received 18 July 2014; revised 10 August 2014; accepted 15 August 2014; accepted article preview online 26 August 2014 ing the body's inflammatory response to this biasing of behavior (Dunn et al, 1991). However, how this is mediated within the brain is currently poorly understood.

Inflammation attenuates novelty exploration across behavioral domains, including physical environments (Spadaro and Dunn, 1990), social interaction with juvenile conspecifics (Bluthe et al, 1992), novel objects (Haba et al, 2012) and preference for novel $v s$ familiar foods (PachecoLópez and Bermúdez-Rattoni, 2011). This range of effects indicates an action on fundamental aspects of novelty processing. Human neuroimaging studies report increased hippocampal activity to novel stimuli (Stern et al, 1996), which is consistent with single-unit (Vinogradova, 2001) and c-Fos studies (Jenkins et al, 2004) in rodents, and also with theoretical models of the hippocampus as a comparator of incoming and previously experienced information (Knight, 1996, Lisman and Grace, 2005). Pro-inflammatory cytokines such as IL-1 can themselves impair hippocampal long-term potentiation (LTP; Katsuki et al, 1990) and synaptic strengthening (Bellinger et al, 1993), as well as spatial (Gibertini, 1996) and contextual (Barrientos et al, 2002) memory, suggesting a plausible locus for the effects of inflammation on novelty preference. Correspondingly, hippocampal stimulation can increase exploratory behavior (Flicker and Geyer, 1982). However, memory-enhancing effects of novelty appear to be mediated via coupling of 
hippocampus with brainstem dopaminergic nuclei (Lisman and Grace, 2005, Duzel et al, 2010). Novel stimuli increase burst firing in reward-sensitive mesolimbic dopaminergic cells (Horvitz et al, 1997; Schultz, 1998) and enhance activity within human substantia nigra (SN; Bunzeck and Duzel, 2006). Further, human SN responses to novelty correlate with a 'novelty bonus' (Wittman et al, 2008) representing the intrinsic reward value of novel stimuli that is hypothesized to drive behavioral exploration (Kakade and Dayan, 2002). A role for dopamine in inflammation-induced attenuation of novelty preference is further suggested by the observation that IL-1-induced reduction in novelty seeking is blocked by the dopamine receptor antagonist sulpiride (Spadaro and Dunn, 1990).

Here, we use typhoid vaccination together with fMRI to dissect the neural basis to effects of inflammation on novelty processing. Typhoid vaccine is an established model of inflammation associated with a threefold increase in proinflammatory cytokines and induction of sickness behaviors $2-3 \mathrm{~h}$ after injection. It is a safe, licensed vaccine routinely administered to people traveling to parts of the world where the likelihood of acquiring typhoid fever is high. Specifically, we tested the prediction that inflammation impairs novelty preference via an action on 'motivational' brainstem nuclei (SN) rich in dopaminergic neurons rather than via an action on hippocampal novelty detection processes.

\section{MATERIALS AND METHODS}

\section{Participants}

Seventeen healthy nonsmokers were recruited from University of Sussex campus advertisements. Volunteers were screened by a consultant psychiatrist (NAH) to ensure that they were healthy, had no previous history of any relevant physical or psychiatric illness, were taking no medication, had no recreational drug use in the previous 6 months and were nonsmokers. Volunteers who had received typhoid vaccine within 3 years or other vaccine within 6 months were excluded. One participant did not complete the second scanning session owing to technical difficulties. Of the remaining 16 participants ( 6 male, mean $27.2 \pm 7.3$ years), 15 were Caucasian and 1 was Indian-Asian. All rated their general health as good, very good or excellent. Participants were advised not to consume caffeinated beverages or alcohol, avoid high-fat meals, and refrain from excessive exercise for $24 \mathrm{~h}$ before testing. They were asked not to take aspirin, ibuprofen or antibiotics for 14 days before testing. Written informed consent was obtained after complete description of the study to the participants. Study procedures were approved by the Brighton East National Research Ethics Committee.

\section{Study Design}

We adopted a randomized, double-blind, crossover repeated measures design in which all participants underwent two separate sessions, an average of 7 days apart (as in previous reports, eg, Harrison et al, 2009). In the first session, participants were randomly assigned to one of two experimental conditions (typhoid vaccine or placebo saline injection). Eight participants received typhoid vaccination in the first session and eight participants received placebo injection. Baseline blood sample was taken and then injections of $0.025 \mathrm{mg}$ Salmonella typhi capsular polysaccharide vaccine (Typhim Vi, Aventis Pasteur MSD, Berkshire, UK) or $0.5 \mathrm{ml}$ of normal saline placebo administered intramuscular (i.m.) into the deltoid muscle. fMRI was performed 2-3 $\mathrm{h}$ after injection in a 60-min scanning session. During each session, participants performed two tasks. The present manuscript focuses on data acquired during an implicit novelty-processing task. Immediately after scanning, a second blood sample was taken ( $3 \mathrm{~h}$ after injection) for cytokine measurement. Body temperature was assessed at baseline and $3 \mathrm{~h}$ with an aural digital thermometer. The second fMRI scanning session was identical except that participants received the alternate injection (ie, typhoid vaccination if they previously received saline and vice versa).

\section{Experimental Model of Inflammation}

We used the S. typhi vaccination model of inflammation that is previously known to induce a low-grade inflammatory cytokine response associated with a two- to threefold increase in peripheral IL-6 levels peaking between 2 and $3 \mathrm{~h}$ (Brydon et al, 2008). S. typhi vaccine $0.025 \mathrm{mg}$ (Typhim Vi, Aventis Pasteur MSD) or $0.5 \mathrm{ml} 0.9 \%$ sodium chloride placebo was administered i.m. into the deltoid muscle by a qualified doctor $(\mathrm{NAH})$. There were no complications of either injection.

\section{Cytokine Analyses}

Separate venepunctures were performed at baseline and $3 \mathrm{~h}$ after injection for both conditions. Blood $(10 \mathrm{ml})$ was drawn into Vacutainer tubes (Becton Dickinson and Company, Franklin Lakes, New Jersey) containing ethylenediaminetetraacetic acid anticoagulant and centrifuged immediately at $1250 \mathrm{~g}$ for $10 \mathrm{~min}$ at room temperature. Plasma was removed, aliquoted, and frozen at $-80^{\circ} \mathrm{C}$ before analysis. Plasma IL-6, IL-1 receptor antagonist (IL-1ra), and tumor necrosis factor alpha $(\mathrm{TNF} \alpha)$ were assessed using high-sensitivity ELISAs (R\&D Systems, Abingdon, UK). The limit of detection of the IL- 6 assay is $0.039 \mathrm{pg} / \mathrm{ml}$, with intra- and interassay coefficients of variation (CVs) of $7.4 \%$ and $7.8 \%$, respectively. The IL-1ra and TNF $\alpha$ assays had detection limits of 0.038 and $6.26 \mathrm{pg} / \mathrm{ml}$ with intra- and interassay CVs of $5.3 \%$ and $8.4 \%$, and $7.8 \%$ and $5.3 \%$, respectively. Cytokine analyses were performed using mixed measures ANOVAs in SPSS 20.0.

\section{Novelty Task}

Immediately before each of the two fMRI scanning sessions, each participant was familiarized to 115 randomly selected gray-scale images (75 outdoor scenes and 40 faces). During this pre-familiarization phase, each image was shown twice for $3 \mathrm{~s}$ and participants were instructed to attend to each image carefully and told they would be asked about them later. Both scanning sessions consisted of two functional runs, in one run the participant was shown novel and familiarized scene images and in the other novel and familiarized face images. All stimuli were of neutral emotional valence. Each run began with presentation of a 'target' outdoor scene or face stimulus that was shown for $4.5 \mathrm{~s}$. Novel, pre-familiarized, and target stimuli were then 
a

Pre-familiarization (outside scanner)
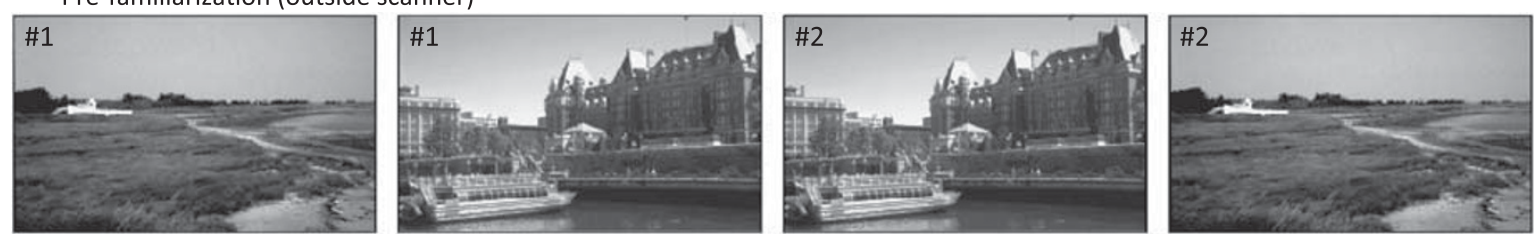

b

Target learning (fMRI)

Incidental processing task (fMRI)
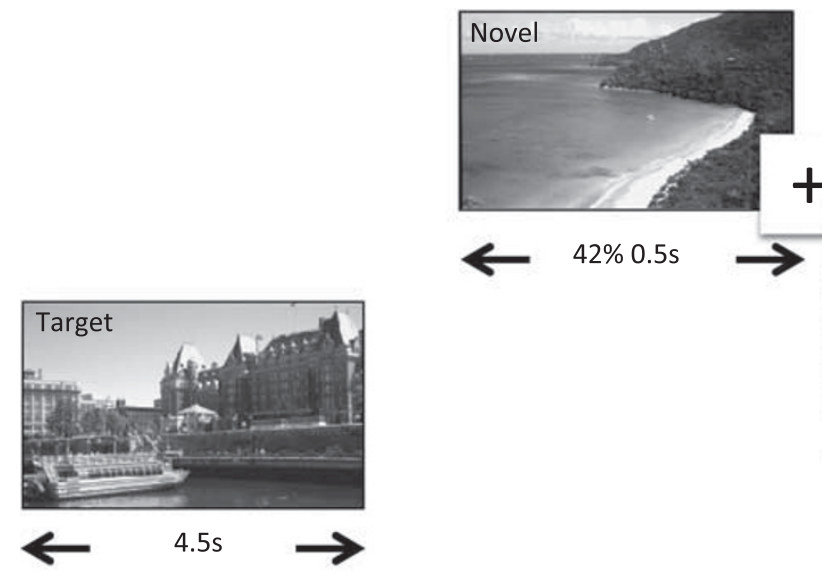

Inter-trial interval 3.4s jittered
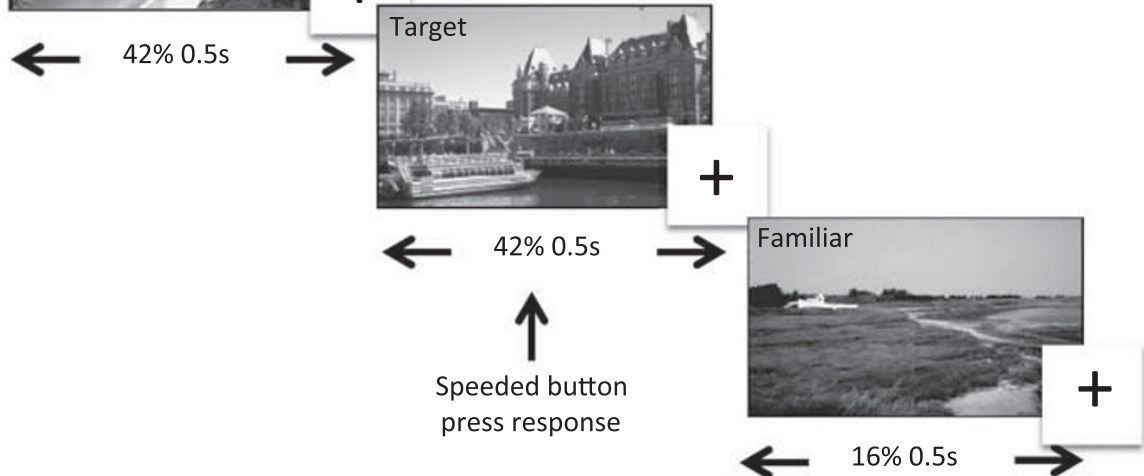

Figure I Task Structure. (a) Participants were pre-familiarized to I I 5 unique images (75 outdoor scenes as illustrated and 40 faces) before each of the two scanning sessions that were completed $2-3 \mathrm{~h}$ after blindly administered typhoid vaccination and saline (placebo) injection. (b) Each scanning session consisted of two separate counterbalanced scanning runs. On one run, participants were shown a single target scene (illustrated on left) followed by randomly presented familiar, target, or novel scenes. Forty-two percent of trials consisted of novel, $42 \%$ familiar, and $16 \%$ target images. Participants made a speeded button press to each presentation of the target stimulus. The second run was identical to the first except that participants were shown target, novel, and familiar face images. Stimuli were presented for $0.5 \mathrm{~s}$ with a jittered (mean $3.4 \mathrm{~s}$ ) intertrial interval.

presented in randomized order, and participants were instructed to respond with a speeded right-handed button press whenever the target image was presented (Figure 1). Targets accounted for $16 \%$ of all trials. Each picture was presented for $0.5 \mathrm{~s}$ with a jittered intertrial interval of $3400 \mathrm{~ms}$. Different sets of images were used for each session counterbalanced across participants.

\section{Image Acquisition}

Gradient-echo single-shot echo planar imaging was used to acquire $\mathrm{T} 2{ }^{\star}$-weighted image volumes on a 1.5 -T Siemens Avanto (Siemens AG Medical Solutions, Erlangen, Germany) scanner equipped with a 12-channel head coil. External restraint was used to minimize head movement. We acquired a total of 480 volumes each with 40 slices (interleaved ascending 2-mm slices with $1-\mathrm{mm}$ interslice gap, echo time $50 \mathrm{~ms}$ : spatial resolution $3 \mathrm{~mm} \times 3 \mathrm{~mm} \times 3$ $\mathrm{mm})$. Slices were tilted $-30^{\circ}$ from the intercommissural plane to reduce orbitofrontal dropout owing to susceptibility artifact from the frontal sinuses (Deichmann et al, 2003). Magnetization transfer (MT) images were also acquired using a 3D gradient-echo sequence (matrix, 192, 192 , and 64 slices; FoV, $200 \times 200 \times 160 \mathrm{~mm}$; spatial resolution, $1.041 .042 .5 \mathrm{~mm}$; $\mathrm{TE}=5 \mathrm{~ms} ; \mathrm{TR}=30 \mathrm{~ms}$; flip angle $=5^{\circ}$ ) to facilitate anatomical identification of the SN and to create an MT template. The MT template was derived by averaging the 16 individual MT images after they were spatially normalized to the standard MNI template supplied in SPM8. High-resolution inversion-recovery echo planar images were also obtained to aid image registration.

\section{Image Analysis}

The fMRI data were analyzed with SPM8 (http://www.fil.ion. ucl.ac.uk/spm). The first 5 volumes were discarded to allow for T1 equilibration. Individual scans were realigned, unwarped, normalized, and spatially smoothed with an 8-mm full-width at half-maximum Gaussian kernel with standard SPM methods. High-pass frequency filter (cutoff $120 \mathrm{~s}$ ) and corrections for auto-correlation between consecutive scans (auto-regressive 1) were applied to the time series. Each event was modeled by a standard synthetic hemodynamic response function at each voxel across the whole brain. Presentations of novel familiar and target images were modeled as separate regressors for both the face and outdoor scene image tasks.

First-level individualized design matrices were estimated in the following manner: effects of task (viewing novel or familiar face or external scene images) were computed on a voxel-wise basis for each participant for both vaccination and placebo conditions in the form of SPMs of discrete contrasts within the general linear model. Subsequent second-level analyses were performed on the SPM contrast 
images with a 2 (novel/familiar $) \times 2($ face/place $) \times 2$ (inflammatory status) factorial ANOVA design to permit formal inferences about population effects.

\section{Regions of Interest}

To address our prior hypotheses of selective effects of inflammation on hippocampal/parahippocampal or SN responses to stimulus novelty, we produced region of interest masks for both regions. The SN mask was custombuilt based on the mean normalized MT image for all participants using MRIcron (http://www.mccauslandcenter.sc.edu/mricro/mricron) and the hippocampal/parahippocampal mask produced using the wfupickatlas (http:// fmri.wfubmc.edu/software/PickAtlas).

\section{Multiple Comparisons}

We used the cluster-extent threshold technique for reporting activated clusters outside of our predefined regions of interest (Slotnick et al, 2003). Specifically, we conducted a MonteCarlo simulation using software written in MATLAB (The MathWorks, Natick, MA; https://www2.bc.edu/ slotnics/ scripts.htm). After running 1000 simulations, we determined that for an individual voxel threshold of $p<0.001$, a clusterextent threshold of 20 contiguous voxels was necessary to correct for multiple comparisons across the whole brain at a significance level of $p<0.05$. Thus, only clusters of activation meeting or exceeding a cluster-extent size of 20 contiguous voxels outside of our predefined regions of interest were considered significantly activated and reported.

\section{Psychophysiological Interaction SN Connectivity Analysis}

To investigate effects of inflammation on changes in SN connectivity to novel stimuli, we first identified psychophysiological interactions (PPIs) of novelty reactivity and functional connectivity with the SN separately in placebo and inflammation conditions. Individual subject data was modeled using fixed-effects GLMs with three condition regressors (novel, familiar, and target conditions), one physiological regressor (first eigenvariate of all voxels within the bilateral SN mask to the contrast novel vs familiar), and a PPI regressor (constructed by multiplying this $\mathrm{SN}$ physiological regressor with the standard regressor of novel $v s$ familiar). The resulting PPI regressors thus modeled differences in SN coupling across the brain as a function of novelty in placebo and inflammation conditions. Using this GLM, individual parameter estimate maps were generated for the contrast of interest: SN connectivity during novel compared with familiar stimuli in placebo and inflammation conditions. Parameter estimate maps for this contrast in both placebo and inflammation conditions were then compared in a second-level paired sample $t$-test.

\section{RESULTS}

\section{Inflammatory Cytokine Responses to Vaccination}

Across participants, typhoid vaccination evoked a robust inflammatory response with an $\sim 250 \%$ increase in plasma
Table I Main Effect of Viewing Novel vs Familiar Objects

\begin{tabular}{|c|c|c|c|c|c|c|}
\hline Side & Region & Coordinates & Z- & $k$ & p- & Corrected \\
\hline$R$ & Hippocampus & {$[40-16-18]$} & 3.50 & 26 & $<0.00$ I & 0.025 \\
\hline$R$ & Parahippocampus & $s[22-38-14]$ & 3.56 & 5 & $<0.001$ & 0.074 \\
\hline$R$ & Mid. frontal gy. & {$\left[\begin{array}{lll}36 & 46 & 36\end{array}\right]$} & 3.98 & 53 & $<0.00$ I & NA \\
\hline $\mathrm{L}$ & Inf. temporal gy. & {$\left[\begin{array}{lll}-42 & 2 & -36\end{array}\right]$} & 3.87 & 34 & $<0.00$ I & NA \\
\hline L & Angular gy. & {$\left[\begin{array}{lll}-38 & -82 & 30\end{array}\right]$} & 3.75 & 33 & $<0.001$ & NA \\
\hline$R$ & Ant. thalamus & {$[6-104]$} & 3.56 & 25 & $<0.001$ & NA \\
\hline
\end{tabular}

Abbreviations: L, left; NA, not applicable; R, right; SVC, small volume corrected.

IL-6 from mean ( \pm SE) $0.70 \pm 0.19 \mathrm{pmol} / \mathrm{l}$ at baseline to $1.74 \pm 0.24 \mathrm{pmol} / \mathrm{l}$ at $3 \mathrm{~h}\left(t_{(15)}=5.20, p<0.001\right)$. The placebo condition evoked a much smaller rise in IL-6 from $0.67 \pm 0.16 \mathrm{pmol} / \mathrm{l}$ at baseline to $1.00 \pm 0.21 \mathrm{pmol} / \mathrm{l}$ at $3 \mathrm{~h}$ $\left(t_{(15)}=1.92, p=0.074\right)$, consistent with a physiological response to experimental stress (Brydon et al, 2004). This was confirmed by a significant treatment (inflammation $v s$ placebo) by sample (baseline and $3 \mathrm{~h}$ ) interaction for IL-6 $\left(\mathrm{F}_{(1,15)}=12.44, p<0.003\right)$. Increases in plasma TNF- $\alpha$ or IL-1 ra did not reach significance, consistent with previous findings (Brydon et al, 2008). No subject had previously received typhoid vaccination, therefore these findings reflect primary immune responses. There was no significant effect of vaccination on core body temperature of participants. Mean change in body temperature after vaccination $-0.06{ }^{\circ} \mathrm{C}$ and placebo $0.12{ }^{\circ} \mathrm{C}$, treatment by sample interaction $\mathrm{F}_{(1,15)}=1.15, p=0.30$.

\section{Responses to Stimulus Novelty}

Perception of novel $v s$ familiar images (main effect of novelty) was associated with significantly greater activity within the right hippocampal region of interest (small volume corrected (SVC) $p<0.025$; Table 1 ). This observation reinforces previously reported findings (Stern et al, 1996). It is noteworthy that hippocampal sensitivity to stimulus novelty was observed in both placebo and experimental inflammation conditions (Figure $2 \mathrm{a}$ and $\mathrm{b}$ ). However, no significant main effect of novelty was observed within the SN region of interest. This result held even when a more permissive uncorrected threshold of $p<0.05$ was adopted, revealing only two voxels on the left side that did not survive SVC $(p=0.308)$.

\section{Effects of Inflammation on Responses to Stimulus Novelty}

To investigate whether inflammation selectively impaired hippocampal or SN responses to stimulus novelty, we next performed the critical inflammation $\times$ stimulus novelty interaction. Although the right hippocampus showed no change in sensitivity to novelty following inflammation, a discrete region within the right parahippocampus did show an attenuation of novelty responses following inflammation (Table 2). Interestingly, this region was adjacent to the area showing a main effect of novelty. Moreover, it was contiguous with a region we previously reported as showing reduced glucose metabolism following typhoid 

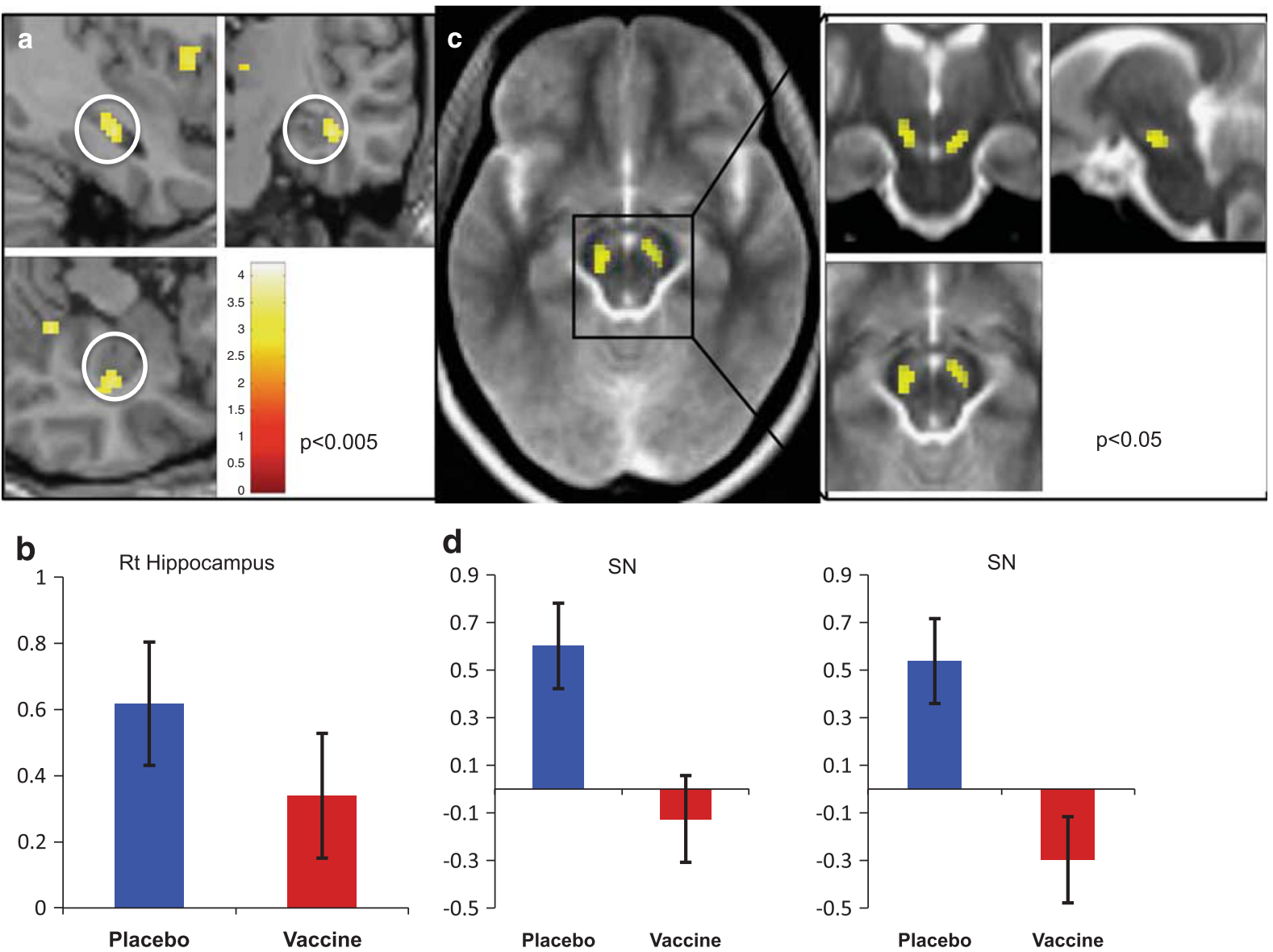

Figure 2 Hippocampal and substantia nigra (SN) responses to novel compared to familiar objects. (a) Right hippocampal region showing increased activation to novel compared with familiar objects (main effect of novelty) Family Wise Error (FWE) corrected $p=0.025$ for hippocampal region of interest. (b) Contrast estimates for the right hippocampal region following placebo (blue) and vaccine (red). (c) SN novelty $\times$ inflammation interaction, FWE corrected $p=0.03$ I (right), $p=0.05$ I (left). (d) Contrast estimates for the right and left SN following placebo (blue) and vaccine (red) demonstrating a significant reduction in $\mathrm{SN}$ responses to novel compared with familiar stimuli bilaterally following inflammation.

vaccine-induced inflammation (Harrison et al, 2014). Crucially, inflammation was associated with a marked reduction in bilateral SN responses to novel compared with familiar stimuli (SVC right $p=0.031$, left $p=0.051$; Table 2, and Figure $2 c$ and $d)$. Interindividual differences in induced IL-6 did not significantly correlate with this change, although did show weak trends ( $p=0.12$ bilaterally) in the anticipated direction, that is, individuals with the greatest IL- 6 response showed the greatest reduction in SN responses to novelty. Targets were correctly identified on $98.4 \%$ of trials, neither target identification nor response time (mean $538.0 \mathrm{~ms}$ ) significantly differed between conditions, $p>0.1$.

\section{Functional Connectivity Analysis}

To characterize these neurophysiological effects more closely, we finally tested the effects of inflammation on novelty-associated changes in $\mathrm{SN}$ connectivity. This analysis at first confirmed the novelty-associated increase in $\mathrm{SN}-$ hippocampus connectivity predicted by the Lisman and Grace, 2005 under basal (placebo) conditions (Table 3). However, this connectivity enhancement was not observed during inflammation. Indeed, paired sample $t$-tests demonstrated that inflammation significantly impaired the no- velty-associated increase in SN-hippocampal connectivity observed under basal conditions (Table 3 ).

Together, our findings indicate that inflammation does not significantly impair hippocampal responses to stimulus novelty yet it does modulate the subsequent processing of this information by the $\mathrm{SN}$ and the functional integration of this information via the functional connectivity of these structures.

\section{DISCUSSION}

Here we show that sub-pyrogenic inflammation selectively impairs human $\mathrm{SN}$ responsivity to novelty, without significantly affecting detection sensitivity to novelty within the hippocampus. Moreover, inflammation also blocked the increased functional connectivity between SN and hippocampus that occurs during novelty processing in noninflammatory states. Together, these data suggest that the impairing effects of inflammation on motivation and behavior (neophilia and novelty exploration) are likely mediated not through an action on hippocampal comparator processes involved in novelty detection, but through actions on the subsequent processing of this information within brainstem dopaminergic structures. Our findings 
Table 2 Interactions between Inflammation and Novelty Processing

\begin{tabular}{|c|c|c|c|c|c|c|}
\hline Side & Region & Coordinates & $\begin{array}{c}\text { Z- } \\
\text { score }\end{array}$ & $k$ & $\begin{array}{c}p- \\
\text { uncor. }\end{array}$ & $\begin{array}{l}\text { Corrected } p \\
\quad(\text { SVC) }\end{array}$ \\
\hline R & Substantia nigra & {$[8-18-14]$} & 2.99 & 37 & $<0.05$ & 0.031 \\
\hline L & Substantia nigra & {$\left[\begin{array}{lll}-10 & -22 & -14\end{array}\right]$} & 2.80 & 51 & $<0.05$ & 0.051 \\
\hline $\mathrm{R}$ & Parahippocampus & {$[28-38-12]$} & 3.74 & 14 & $<0.001$ & 0.041 \\
\hline L & Hippocampus & {$\left[\begin{array}{lll}-20 & -28 & -12\end{array}\right]$} & 3.44 & 15 & $<0.001$ & 0.096 \\
\hline $\mathrm{R}$ & Fusiform gy. & {$\left[\begin{array}{lll}36 & -48 & -22\end{array}\right]$} & 4.37 & 133 & $<0.001$ & NA \\
\hline L & Mid. temporal gy. & {$\left[\begin{array}{lll}-52 & 0 & -34\end{array}\right]$} & 4.07 & 33 & $<0.001$ & NA \\
\hline $\mathrm{R}$ & Amygdala & {$\left[\begin{array}{lll}18 & -2 & -20\end{array}\right]$} & 3.74 & 123 & $<0.001$ & NA \\
\hline L & Fusiform gy. & {$\left[\begin{array}{lll}-38 & -34 & -20\end{array}\right]$} & 3.86 & 50 & $<0.00$ I & NA \\
\hline R & Inf. occipital gy. & {$[46-70-10]$} & 3.74 & 42 & $<0.001$ & NA \\
\hline L & Post-central gy. & {$\left[\begin{array}{lll}-48 & -38 & 60\end{array}\right]$} & 3.74 & 51 & $<0.001$ & NA \\
\hline $\mathrm{R}$ & Amygdala & {$\left[\begin{array}{lll}18 & -2 & -20\end{array}\right]$} & 3.74 & 123 & $<0.001$ & NA \\
\hline $\mathrm{R}$ & dorsal ACC & {$\left[\begin{array}{lll}8 & 32 & 52\end{array}\right]$} & 3.67 & 46 & $<0.001$ & NA \\
\hline L & Sup. temporal sul. & {$\left[\begin{array}{lll}-52 & -40\end{array}\right]$} & 3.65 & 29 & $<0.001$ & NA \\
\hline
\end{tabular}

provide empirical support for predictions arising from the novelty-related motivation of anticipation and exploration by dopamine (NOMAD) theory, which proposes that motivating effects of novelty are mediated via neuromodulatory dopaminergic pathways originating in brainstem (Duzel et al, 2010).

Converging evidence from humans, nonhuman primates, and rodents points to a specialized brain system for the detection of novelty, centered around the hippocampus and medial temporal lobe (MTL) memory system (Ranganath and Rainer, 2003). Both hippocampus and adjacent MTL structures respond robustly to novel stimuli (Stern et al, 1996; Bunzeck and Duzel, 2006; Howard et al, 2011). Hippocampal stimulation also increases exploratory behavior in a similar manner to novelty itself (Flicker and Geyer, 1982; Yang and Mogenson, 1987), an action that can be blocked by lesions to the ventral subiculum subregion of the hippocampus (Legault and Wise, 2001). However, human and animal studies also show that dopaminergic neuromodulation, originating from midbrain dopaminergic nuclei ( $\mathrm{SN}$ and ventral tegmental area), enhance hippocampal synaptic plasticity in response to novel events, engendering a motivationally energizing effect on actions. Correspondingly, novel stimuli elicit increased activity not only within hippocampus but also SN and ventral striatum, regions implicated in generating motivational drive (Bunzeck and Duzel, 2006). A hippocampal-VTA/SN circuit is also proposed to control entry of information into long-term memory. In this model, sensory inputs enter hippocampus CA1 from entorhinal cortex. Information regarding detected novelty is outputted to both the midbrain (SN/VTA) and ventral striatum, where it is contextually integrated with other motivational goals. These trigger VTA projection neurons to release dopamine onto D1/D5 receptors at hippocampus CA1 synapses to enhance LTP and learning (Lisman and Grace, 2005). This specific translation of novelty detection into prospective effects on memory fits within a wider context of dopamine-mediated regulation of motivational behavior, including reward seeking (Pessiglione et al, 2006), addiction (Hyman et al, 2006), drive (Robbins and Everitt, 2007), and incentive motivation (Berridge, 2007). Moreover, the motivational effects of
Table 3 Effects of Novelty on SN Functional Connectivity

\begin{tabular}{|c|c|c|c|c|c|c|}
\hline Side & Region & Coordinates & $\begin{array}{c}\text { Z- } \\
\text { score }\end{array}$ & $k$ & $\begin{array}{c}\text { p- } \\
\text { uncor. }\end{array}$ & $\begin{array}{l}\text { Corrected } p \\
\quad(\text { SVC) }\end{array}$ \\
\hline \multicolumn{7}{|c|}{ Placebo } \\
\hline $\mathrm{L}$ & Hippocampus & {$\left[\begin{array}{lll}-24 & -24 & -10\end{array}\right]$} & 3.99 & 24 & $<0.001$ & 0.024 \\
\hline $\mathrm{R}$ & Hippocampus & {$[20-12-20]$} & 3.94 & 59 & $<0.001$ & 0.029 \\
\hline $\mathrm{L}$ & Retrosplenium & {$\left[\begin{array}{lll}-16 & -50 & 8\end{array}\right]$} & 3.64 & 23 & $<0.001$ & NA \\
\hline$L$ & Retrosplenium & {$\left[\begin{array}{lll}-4 & -58 & 10\end{array}\right]$} & 3.55 & 53 & $<0.001$ & NA \\
\hline \multicolumn{7}{|c|}{ Inflammation } \\
\hline R & Mid. temporal g. & {$\left[\begin{array}{lll}32 & 8 & -34\end{array}\right]$} & 3.92 & 37 & $<0.001$ & NA \\
\hline \multicolumn{7}{|c|}{ Effects of inflammation (inflammation-placebo) } \\
\hline $\mathrm{R}$ & Hippocampus & {$[24-22-20]$} & 4.17 & 23 & 0.001 & 0.014 \\
\hline $\mathrm{L}$ & Hippocampus & {$\left[\begin{array}{lll}-12 & -34 & -12\end{array}\right]$} & 3.63 & 26 & 0.001 & 0.184 \\
\hline
\end{tabular}

dopamine may manifest increased response vigor (Niv et al, 2007), which may in turn offset the increased risk associated with exploratory exposure. The 'NOMAD' concept proposed by Duzel et al, 2010 attempts to unite these aspects of dopamine function in a model that informs our understanding of memory, ageing, and neurodegeneration. Correspondingly, our data provide further insight into the perturbation of adaptive responses to novelty by inflammatory mechanisms implicated in neuropathological processes, including progression of dementia (Perry et al, 2007) and depression.

Typically, inflammation is thought to impair memory through direct effects on hippocampal function (Yirmiya and Goshen, 2011). Our data now indicate that consequences of inflammation on the encoding and consolidation of new memories may also be mediated via indirect actions on the ascending arm of the hippocampal-VTA loop. Dopamine release within the hippocampus, from neurons originating in SN, enhances LTP and learning (Lisman and Grace, 2005). Our findings not only suggest that this interaction is compromised by inflammation but also can inform current controversies about how inflammation mediates memory impairment. Paradoxical effects of inflammation have been observed, for example, induction of low-grade inflammation (with lipopolysaccharide) in mice has been shown to enhance simple spatial discrimination learning on a T-maze side-discrimination task when one arm was always rewarded, yet impair it when the correct arm was rewarded on only $50 \%$ of trials (Sanderson et al, 2009). These observations require an alternative to a purely hippocampus-dependent account of inflammationinduced learning and memory deficits, and highlight the potential role of dopaminergic motivational mechanisms. Our data extend such observations to humans, presenting evidence for intact hippocampal novelty detection yet compromised dopaminergic signaling of stimulus novelty (plausibly reflecting motivational salience) during peripheral states of inflammation.

Interestingly, our findings of reduced $\mathrm{SN}$ novelty responses were observed in response to a relatively mild inflammatory challenge, suggesting particular sensitivity of novelty-processing mechanisms to inflammatory status. This finding 
accords well with recent rodent data demonstrating a marked reduction in responses to novel objects even after doses of intraperitoneal LPS that were insufficient to impair food intake, immobility on the forced swim test, or social interaction (Haba et al, 2012). Low-dose Staphylococcus aureus enterotoxin- $\mathrm{B}$ (a superantigen that induces T-cell activation and IL-2, interferon, and TNF release) also been shown to impair food intake in a novel but not familiar context without changing mobility or weight, suggesting a discrete effect on novelty responses unconfounded by illness anorexia (Kawashima and Kusnecov, 2002). Enhanced neophobia in this model was also reported towards nonedible inanimate objects reflecting an increase in neophobic behavior more generally (Kawashima and Kusnecov, 2002). Finally, temporal responses to LPS challenge have demonstrated impaired novelty responses for up to $24 \mathrm{~h}$, long after changes in locomotor activity have returned to baseline, again supporting the suggestion that impaired novelty preference is one of the most vulnerable behaviors to peripheral inflammatory challenge (Haba et al, 2012). They also support the concept of a 'behavioral immune system' designated not just to fight pathogens but also bias behavior to avoid the risk of infection (Schaller and Park, 2011).

Why peripheral inflammation should have such a profound effect on responses to stimulus novelty is currently unclear. However, may be usefully informed by consideration of the potentially adaptive advantages of sickness behaviors more generally. Immune responses demand substantial energy investment (Straub et al, 2010) and typically occur when food intake is reduced perhaps to restrict further exposure to a common source of infection or alternately restrict micronutrient availability required by some pathogens to replicate. It is perhaps unsurprising, therefore, that energy intense processes such as foraging and hunting, reproduction, and lactation are compromised during sickness responses to infection (Pacheco-López and Bermúdez-Rattoni, 2011). Increase in c-Fos expression in cortico-limbic structures such as the insula and amygdala following inflammatory challenge have been used to propose that immune-mediated enhancement of emotionality potentiates innate avoidant behavior reducing unnecessary danger when body energy resources are diverted (Pacheco-López and Bermúdez-Rattoni, 2011). However, long-lasting effects of immunoactivation on novelty responding have been demonstrated even in the absence of associated anxiety-like behaviors measured in open-field and elevated plus-maze tests, suggesting that they are unlikely to represent nonspecific anxiety responses (Haba et al, 2012). Our current data instead suggest a more discrete mechanism in which inflammation selectively impairs SN responses to novelty, a region rich in dopaminergic neurons that have been proposed to drive exploratory behavior with less beneficial effects on memory formation as a side effect.

We have also previously shown that change in response time on a Stroop task following typhoid vaccination correlated with increases in circulating IL-6 levels. Further, this correlated with reactivity of the SN (Brydon et al, 2008), suggesting a sensitivity of human SN to systemic inflammation. For humans, there is of course more to novelty than just foraging. Indeed, it has been argued that an interest in novelty and engagement of novelty-seeking behaviors 'the lure of the unknown' is a driving force behind many of mankind's great discoveries (Knutson and Cooper, 2006). Our identification of a discrete action of inflammation on human SN responses to novelty offers a novel experimental model in which to further explore this.

\section{FUNDING AND DISCLOSURE}

The authors declare no conflict of interest.

\section{ACKNOWLEDGEMENTS}

This research was supported by Wellcome Trust Intermediate Clinical Fellowships awarded to NAH and VV, and European Research Council support to HDC. Neither the Wellcome Trust nor the European Research Council had any role in the design of the study, collection and analysis of data or decision to publish the data.

\section{REFERENCES}

Barrientos RM, Higgins EA, Sprunger DB, Watkins LR, Rudy JW, Maier SF (2002). Memory for context is impaired by a post context exposure injection of interleukin-1 beta into dorsal hippocampus. Behav Brain Res 134: 291-298.

Bellinger FP, Madamba S, Siggins GR (1993). Interleukin 1 beta inhibits synaptic strength and long-term potentiation in the rat CA1 hippocampus. Brain Res 628: 227-234.

Berlyne DE (1955). The arousal and satiation of perceptual curiosity in the rat. J Comp Physiol Psychol 48: 238-246.

Berridge KC (2007). The debate over dopamine's role in reward: the case for incentive salience. Psychopharmacology 191: 391-431.

Bluthe RM, Crestani F, Kelley KW, Dantzer R (1992). Mechanisms of the behavioral effects of interleukin-1: role of prostaglandins and CRF. Ann N Y Acad Sci 650: 268-275.

Brydon L, Edwards S, Mohamed-Ali V, Steptoe A (2004). Socioeconomic status and stress-induced increases in interleukin-6. Brain Behav Immun 18: 281-290.

Brydon L, Harrison NA, Walker C, Steptoe A, Critchley HD (2008). Peripheral inflammation is associated with altered substantia nigra activity and psychomotor slowing in humans. Biol Psychiatry 63: 1022-1029.

Bunzeck N, Duzel E (2006). Absolute coding of stimulus novelty in the human substantia nigra/VTA. Neuron 51: 369-379.

Deichmann R, Gottfried JA, Hutton C, Turner R (2003). Optimized EPI for fMRI studies of the orbitofrontal cortex. Neuroimage 19: 430-441.

Dunn AJ, Antoon M, Chapman Y (1991). Reduction of exploratory behavior by intraperitoneal injection of interleukin-1 involves brain corticotropin-releasing factor. Brain Res Bull 26: 539-542.

Duzel E, Bunzeck N, Guitart-Masip M, Duzel S (2010). Noveltyrelated Motivation of Anticipation and exploration by Dopamine (NOMAD): Implications for healthy ageing. Neurosci Biobehav Rev 34: 660-669.

Flicker C, Geyer MA (1982). Behavior during hippocampal microinfusions. I. Norepinephrine and diversive exploration. Brain Res Rev 4: 79-103.

Gibertini M (1996). IL1 beta impairs relational but not procedural rodent learning in a water maze task. Adv Exp Med Biol 402: 207-217.

Haba R, Shintani N, Onaka Y, Wang H, Takenaga R, Hayata A et al (2012). Lipopolysaccharide affects exploratory behaviors towards novel objects by impairing cognition and/or motivation in mice: possible role of activation of the central amygdala. Behav Brain Res 228: 423-431. 
Harrison NA, Brydon L, Walker C, Gray MA, Steptoe A, Dolan RJ et al (2009). Neural origins of human sickness in interoceptive responses to inflammation. Biol Psychiatry 66: 415-422.

Harrison NA, Doeller CF, Voon V, Burgess N, Critchley HD (2014). Peripheral inflammation acutely impairs human spatial memory via actions on medial temporal lobe glucose metabolism. Biol Psychiatry 76: 585-593.

Hart BL (1988). Biological basis of the behavior of sick animals. Neurosci Biobehav Rev 12: 123-137.

Horvitz JC, Stewart T, Jacobs BL (1997). Burst activity of ventral tegmental dopamine neurons is elicited by sensory stimuli in the awake cat. Brain Res 759: 251-258.

Howard LR, Kumaran D, Ólafsdóttir HF, Spiers HF (2011). Double dissociation between hippocampal and parahippocampal responses to object-background context and scene novelty. J Neurosci 31: 5253-5261.

Hyman SE, Malenka RC, Nestler EJ (2006). Neural mechanisms of addiction: the role of reward-related learning and memory. Annu Rev Neurosci 29: 565-598.

Jenkins TA, Amin E, Pearce JM, Brown MW, Aggleton JP (2004). Novel spatial arrangements of familiar visual stimuli promote activity in the rat hippocampal formation but not the parahippocampal cortices: a c-fos expression study. Neuroscience 124: 43-52.

Kakade S, Dayan P (2002). Dopamine: generalization and bonuses. Neural Netw 15: 549-559.

Katsuki H, Nakai S, Hirai Y, Akaji K, Kiso Y, Satoh M (1990). Interleukin- $1 \beta$ inhibits long-term potentiation in the CA3 region of mouse hippocampal slices. Eur J Pharmacol 181: 323-332.

Kawashima N, Kusnecov AW (2002). Effects of staphylococcal enterotoxin A on pituitary-adrenal activation and neophobic behavior in the C57BL/6 mouse. J Neuroimmunol 123: 41-49.

Knight RT (1996). Contribution of human hippocampal region to novelty detection. Nature 383: 256-259.

Knutson B, Cooper JC (2006). The lure of the unknown. Neuron 51: 280-282.

Kusnecov AW, Liang R, Shurin G (1999). T-lymphocyte activation increases hypothalamic and amygdaloid expression of CRH mRNA and emotional reactivity to novelty. J Neurosci 19: 4533-4543.

Legault M, Wise RA (2001). Novelty-evoked elevations of nucleus accumbens dopamine: dependence on impulse flow from the ventral subiculum and glutamatergic neurotransmission in the ventral tegmental area. Eur J Neurosci 13: 819-828.

Lima SL, Dill LM (1990). Behavioral decisions made under the risk of predation: a review and prospectus. Can J Zool 68: 619-640.

Lisman JE, Grace AA (2005). The hippocampal-VTA loop: controlling the entry of information into long-term memory. Neuron 46: 703-713.

Niv Y, Daw ND, Joel D, Dayan P (2007). Tonic dopamine: opportunity costs and the control of response vigor. Psychopharmacology 191: 507-520.

Pacheco-López G, Bermúdez-Rattoni F (2011). Brain-immune interactions and the neural basis of disease-avoidant ingestive behavior. Phil Trans R Soc B 366: 3389-3405.

Panksepp J (1998). Affective Neuroscience: The Foundations of Human and Animal Emotions. 1st edn. Oxford University Press: New York, NY, USA.
Perry VH, Cunningham C, Holmes C (2007). Systemic infections and inflammation affect chronic neurodegeneration. Nat Rev Immunol 7: 161-167.

Pessiglione M, Seymour B, Flandin G, Dolan RJ, Frith CD (2006). Dopamine-dependent prediction errors underpin reward-seeking behavior in humans. Nature 442: 1042-1045.

Ranganath C, Rainer G (2003). Neural mechanisms for detecting and remembering novel events. Nature Rev Neurosci 4: 193-202.

Robbins TW, Everitt BJ (2007). A role for mesencephalic dopamine in activation: commentary on Berridge (2006). Psychopharmacology 191: 433-437.

Sanderson DJ, Cunningham C, Deacon RM, Bannerman DM, Perry VH, Rawlins JN (2009). A double dissociation between the effects of sub-pyrogenic systemic inflammation and hippocampal lesions on learning. Behav Brain Res 201: 103-111.

Schaller M, Park JH (2011). The behavioral immune system (and why it matters). Curr Dir Psychol Sci 20: 99-103.

Schultz W (1998). Predictive reward signal of dopamine neurons. J Neurophys 80: 1-27.

Slotnick SD, Moo LR, Segal JB, Hart J (2003). Distinct prefrontal cortex activity associated with item memory and source memory for visual shapes. Cogn Brain Res 17: 75-82.

Spadaro F, Dunn AJ (1990). Intracerebroventricular administration of interleukin-1 to mice alters investigation of stimuli in a novel environment. Brain Behav Immun 4: 308-322.

Stern CE, Corkin S, Gonzalez RG, Guimaraes AR, Baker JR, Jennings PJ et al (1996). The hippocampal formation participates in novel picture encoding: Evidence from functional magnetic resonance imaging. Proc Natl Acad Sci USA 93: 8660-8665.

Straub RH, Cutolo M, Buttgereit F, Pongratz G (2010). Energy regulation and neuroendocrine-immune control in chronic inflammatory diseases. J Intern Med 267: 543-560.

Vinogradova OS (2001). Hippocampus as comparator: role of the two input and two output systems of the hippocampus in selection and registration of information. Hippocampus 11: 578-598.

Wittmann BC, Daw ND, Seymour B, Dolan RJ (2008). Striatal activity underlies novelty-based choice in humans. Neuron 58: 967-973.

Yang CR, Mogenson GJ (1987). Hippocampal signal transmission to the pedunculopontine nucleus and its regulation by dopamine D2 receptors in the nucleus accumbens: An electrophysiological and behavioral study. Neuroscience 23: 1041-1055.

Yirmiya R, Goshen I (2011). Immune modulation of learning, memory, neural plasticity and neurogenesis. Brain Behav Immun 25: 181-213.

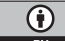

This work is licensed under a Creative Commons Attribution 4.0 International License. The images or other third party material in this article are included in the article's Creative Commons license, unless indicated otherwise in the credit line; if the material is not included under the Creative Commons license, users will need to obtain permission from the license holder to reproduce the material. To view a copy of this license, visit http://creativecommons.org/ licenses/by/4.0/ 